\title{
PENSANDO LA EVIDENCIA ARQUEOLÓGICA DESDE LA GEOGRAFÍA: PROPUESTA METODOLÓGICA PARA LA IDENTIFICACIÓN DE LAS TERRITORIALIDADES DE LAS POBLACIONES ANTIGUAS DE COSTA RICA
}

\section{THINKING ABOUT THE ARCHEOLOGICAL EVIDENCE FROM GEOGRAPHY: METHODOLOGICAL PURPOSE FOR THE IDENTIFICATION OF THE TERRITORIALITIES OF COSTA RICAN ANCIENT POPULATIONS}

\section{RESUMO}

El presente artículo tiene como objetivo principal justificar la utilidad de trabajar de manera interdisciplinaria entre la Geografía y la Arqueología, esto como medio para aproximarnos a comprender parte de las realidades sociales de las poblaciones antiguas. Lo anterior se expone a partir de una propuesta metodológica para la identificación de las territorialidades de las poblaciones que habitaron lo que hoy se conoce como Costa Rica, según el sitio arqueológico de interés, siendo un insumo significativo para la gestión comunitaria del Patrimonio Arqueológico en pro de su conservación y preservación como un producto turístico.

Palavras-chave: Geografía del Turismo; Arqueología; Territorialidad; Patrimonio Arqueológico; Puesta en valor turístico.

\section{ABSTRACT}

The main objective of the present article is to justify the practicality of interdisciplinary work between the fields of Geography and Archeology as an approach towards comprehending the realities of ancient populations. This is set out on the basis of a methodological proposal to identify the territorialities of ancient settlers that inhabited the territories of what today is known as Costa Rica, according to the archeological site of interest, as a significant contribution towards community management of Archeological Patrimony aiming towards its conservation and preservation as a touristic product.

Keywords: Tourism Geography; Archeology; Territoriality; Archaeological Heritage; Tourism value enhancement.

\section{Samira Jalet Quesada ${ }^{a}$}

a Universidad Nacional de Costa Rica (UNA), Heredia, Costa Rica

DOI: 10.12957/geouerj.2020.51271

Correpondência: jaletquesada@gmail.com

Recebido em: 26 mai. 2020

Aceito em: 25 set. 2020 


\section{INTRODUCCIÓN}

El concepto de Patrimonio Arqueológico es definido por Arias (1999) como la cultura material dejada por las poblaciones antiguas en un determinado espacio. Mismo que transformaron en territorio a partir del desarrollo de diversas prácticas espaciales que permitieron la continuidad de la humanidad en diversas manifestaciones. Además, Arias y Chávez (1999) mencionan que es a partir de los artefactos, ecofactos, estructuras arquitectónicas, modificación al ambiente, entre otros, que se conforma la cultura material, por lo que son el producto de acciones necesarias para la sobrevivencia y la convivencia humana.

Según la Organización de las Naciones Unidas para la Educación, la Ciencia y la Cultura (UNESCO) (2006), el Patrimonio Arqueológico forma parte del Patrimonio Cultural, debido a que refleja bienes materiales, ya sean monumentos, conjuntos y/o lugares que heredamos de nuestros antepasados, los cuales representan un valor único y excepcional del cual todos y todas tenemos el derecho de disfrutar en el presente y se debe de resguardar para el goce de las futuras generaciones. Por lo tanto, la importancia de su protección, según Baldeón (2002) y Arroyo (2017), radica en que es un recurso frágil, delicadamente vulnerable y no renovable, por lo que su alteración provoca la destrucción para siempre de los datos que puedan permitir reconstruir parte de la historia de la humanidad.

Debido a lo anterior, el objetivo principal del presente artículo, es presentar una propuesta metodológica la cual permita identificar las posibles territorialidades -por sitio arqueológico- de las poblaciones antiguas que habitaron el espacio actual costarricense. Lo anterior con el fin de que funcione como un insumo el cual pueda ser ejecutado desde la Geografía del Turismo para la gestión comunitaria del Patrimonio Arqueológico en coordinación con el Museo Nacional de Costa Rica, ente estatal encargado de velar por los bienes patrimoniales arqueológicos. La misma, se focaliza en el análisis espacial de elementos físico-geográficos presentes en el sitio arqueológico, así como del análisis de la vinculación de algunos conceptos claves desde la Geografía con algunos indicadores arqueológicos.

Por lo tanto, la estructura del presente artículo incluye tres apartados. El primero presenta una síntesis de la definición de los conceptos de espacio, región, territorio, paisaje y lugar, sentando la base a partir de la cual se construyen las categorías analíticas geográficas presentadas en el tercer apartado. Asimismo, se ejemplifican de manera concreta la implementación de dichos conceptos dentro del análisis de la evidencia arqueológica, esto con el fin de demostrar su utilidad para la identificación de las territorialidades de las poblaciones antiguas. En el segundo, se justifica conceptualmente el abordaje de la gestión comunitaria para el resguardo del Patrimonio Arqueológico considerando como base a la Geografía del Turismo. Por último, se expone la propuesta metodológica. 


\section{ESPACIO, REGIÓN, TERRITORIO, PAISAJE Y LUGAR: CONCEPTOS CLAVES DESDE LA GEOGRAFÍA}

Para Côrrea (2000a), la Geografía como Ciencia Social analiza parte de la realidad social a partir de la implementación integrada de cinco conceptos claves: espacio, región, territorio, paisaje y lugar. Esto, debido a que permiten identificar las acciones y transformaciones que ha desarrollado la sociedad en la superficie terrestre. Por lo tanto, a continuación se definen de manera puntual dichos conceptos y se ejemplifican a partir de la evidencia presente en un sitio arqueológico localizado en Costa Rica.

Iniciando con el concepto de espacio, según Santos (1990), el mismo es un producto de la sociedad, dinámico y cambiante, resultado de las relaciones sociales, económicas, políticas y ambientales, a partir de las necesidades e intereses que aclaman las sociedades. Por otra parte, Côrrea (2000a) identifica algunas prácticas espaciales que contribuyen a la construcción del espacio social, debido a que son acciones que se ejecutan para organizarlo, como bien menciona que las mismas "son medios efectivos a través de los cuales se gestiona el territorio, esto es, la administración y el control de la organización espacial en su existencia y reproducción" ${ }^{1}$ (CÔRREA, 2000a, p. 35, traducción propia). Destacando tres prácticas espaciales mencionadas por Côrrea (2000a), se encuentran:

a. Selectividad espacial: de lo propuesto por Côrrea (2000a), en relación a las poblaciones antiguas, esta práctica espacial permite analizar que fueron conscientes en relación al espacio o espacios que eligieron para vivir o transitar, pensando en que este les proveyera seguridad, alimento y/o bienestar.

b. Fragmentación espacial²: según Côrrea (2000a), hace referencia a que una sociedad al ejercer control político en un espacio determinado provocará que el mismo se fragmente en unidades territoriales, siendo las mismas porciones del espacio las cuales responden al desarrollo de actividades en específico. Esto se refleja en la evidencia arqueológica del sitio Monumento Nacional Guayabo (C-362 MNG) (ver imagen 1 y 2), localizado en Turrialba, Cartago, Costa Rica. La misma, según Aguilar (1972) y (Fonseca, 1992 apud Herrera y Arias, 2016), permite inferir que el sitio responde a una organización socio-política jerarquizada, en donde el ejercicio del poder lo pudo realizar una élite gobernante localizada en una unidad territorial. Esto les permitía controlar los aspectos relacionados al ámbito político, social, religioso, económico y ambiental. Por lo tanto, debió de existir una especialización por parte de la comunidad, como: artesanos/as dedicados/as al trabajo en cerámica, textiles, lítica, encargados/as religiosos/as, entre otros, localizadas en sus respectivas unidades territoriales.

\footnotetext{
${ }^{1}$ Texto original: "são meios efetivos através dos quais objetiva-se a gestão do territorio, isto é, a administração e o controle da organização espacial em sua existência e reprodução".

2 Texto original: "fragmentação-remembramento espacial".
} 
Imagen 1. Sitio Monumento Nacional Guayabo (C-362 MNG). Fuente: Propia. Fecha: 17 de mayo del 2017. Lugar: Turrialba, Cartago, Costa Rica.

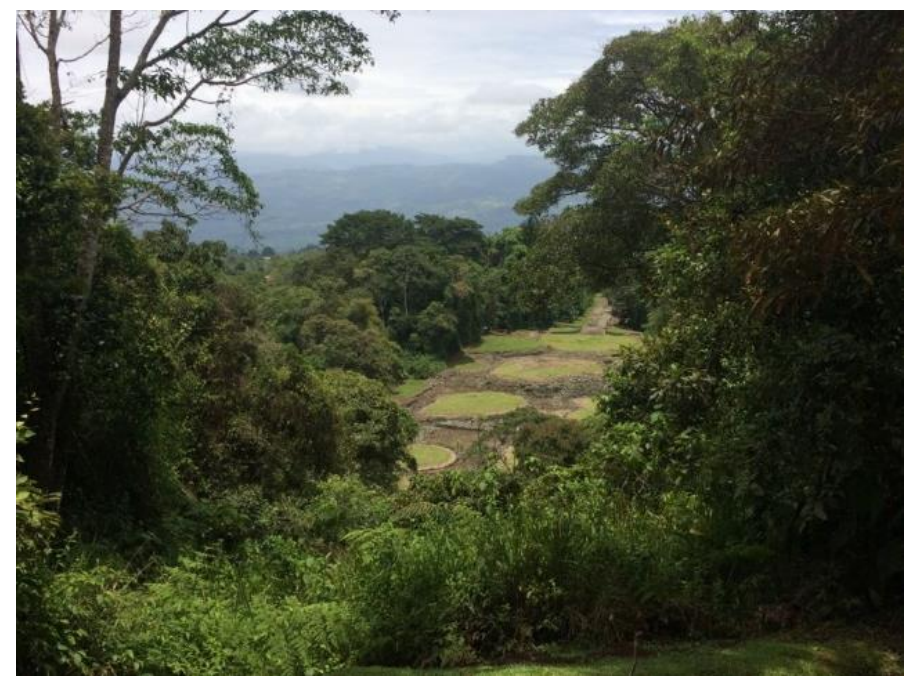

Imagen 2. Localización del sitio Monumento Nacional Guayabo (C-362 MNG). Fuente: Herrera y Arias, 2016, p. 178.
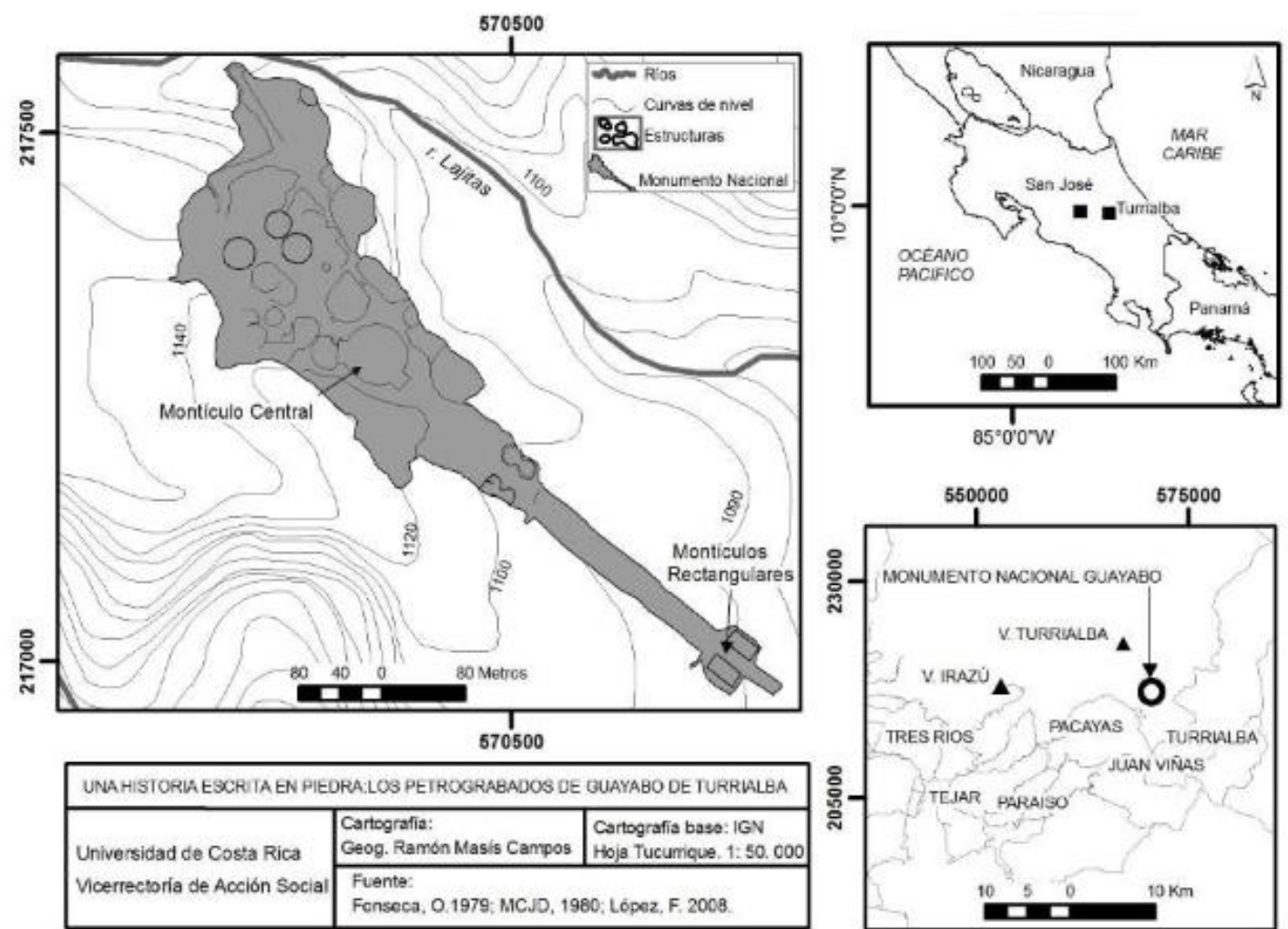

c. Región productora: la misma, según Côrrea (2000a), explica la gestión territorial por medio de las prácticas que se han consolidado colectivamente y han sobrevivido de generación en generación. Esto se puede analizar en la evidencia arqueológica considerando la variable espacial y temporal, debido a que es viable identificar una práctica espacial determinada y su posible continuidad. Ejemplo de esto lo demuestran los datos arqueológicos del sitio Nacascolo (G-89 Na), localizado 
en Bahía Culebra, Guanacaste, Costa Rica (ver imagen 3). Para el mismo, según Gutiérrez (1997), se ha inferido que las poblaciones antiguas que lo habitaron se dedicaron a actividades pesqueras, esto por medio de los datos que se han recopilado de los concheros o basureros, siendo estos definidos como "una acumulación antropogénica intencional de concha"3 (BALBO ET AL, 2011, p. 147 apud BEOVIDE, 2013, p. 137, traducción propia). Según la misma autora, de los mismos, se extrajo información que permite inferir que los recursos marinos se incluyeron en sus dietas y formaron parte de sus modos de vida por un lapso aproximado de 1250 años.

Imagen 3. Localización del sitio Nacascolo (G-89 Na). Fuente: Gutiérrez, 1997, p. 161.

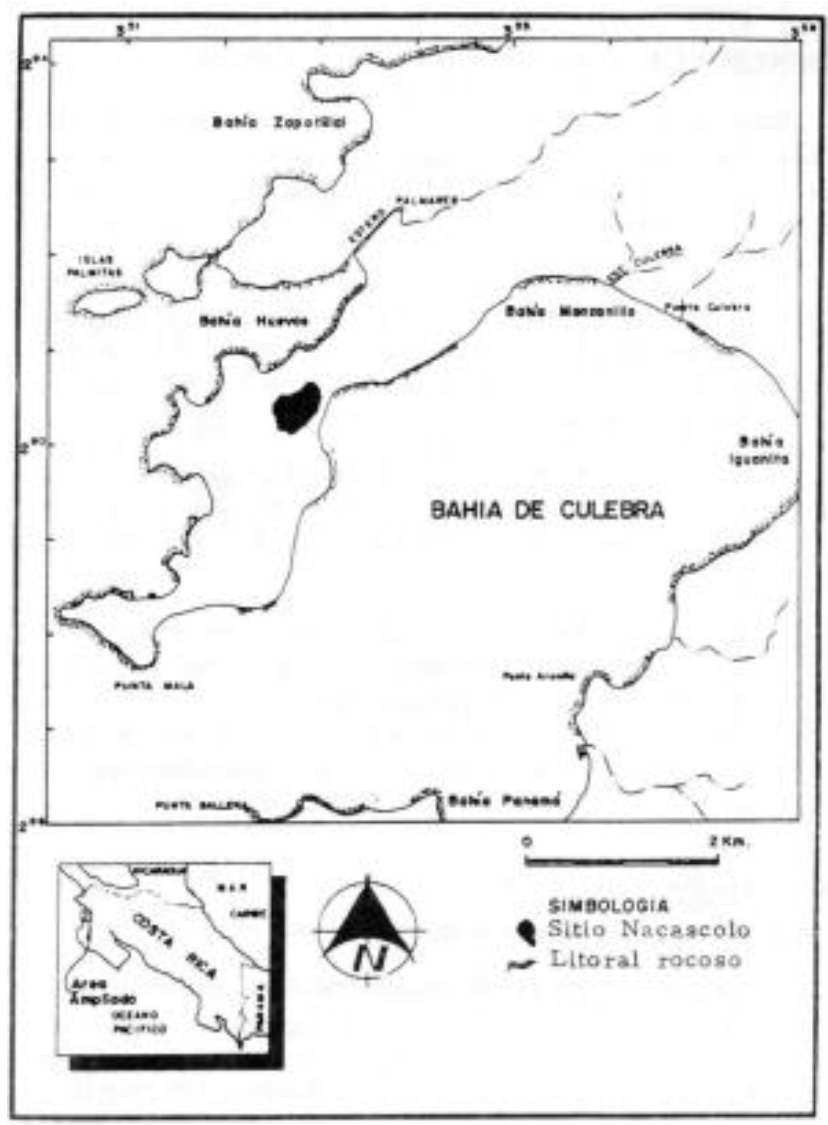

Ahora bien, como segundo concepto se encuentra la región, definiéndose la misma a partir de Côrrea (2000b) y Haesbaert (1999) como un espacio el cual se delimita a partir de la presencia de un conjunto de características particulares que reflejan una coherencia y articulación funcional que lo hacen diferenciable. Por lo tanto, existen diversas posibilidades de tipos de regiones, dependiendo de las necesidades e intereses de conformarlas, destacando que dentro de la arqueología el concepto de región es aplicado y es un insumo básico para tratar de comprender las relaciones de las poblaciones antiguas. Esto debido, por ejemplo, a que

\footnotetext{
${ }^{3}$ Texto original: "as intentional anthropogenic shell accumulations"
} 
sí se conforman regiones arqueológicas a partir de sus semejanzas "en la cerámica, en los asentamientos y en los enterramientos" ${ }^{\prime 4}$ (CORRALES, 2000, p. 16, traducción propia), se podrán apreciar posibles relaciones de intercambio, ya sea a nivel comercial o incluso el intercambio de ideas.

En el caso de Costa Rica, se han establecido tres grandes regiones arqueológicas (ver imagen 4), presentándolas Corrales (2001) como:

a. Región Arqueológica Gran Nicoya: incluye parte del Pacífico Sur de Nicaragua y se denomina Subregión Sur o Guanacaste al espacio que abarca la actual provincia de Guanacaste y el Golfo de Nicoya, incluyendo el noroeste de Puntarenas.

b. Región Arqueológica Central: se divide en tres subregiones; Subregión Central-Pacífica (Valle Central y Pacífico Central), Subregión Caribe (Valle de Turrialba y Llanuras del Caribe Central) y Subregión Llanuras del Norte.

c. Región Arqueológica Gran Chiriquí: la cual abarca parte del oeste de Panamá (Chiriquí), y al espacio ubicado dentro de Costa Rica se le denomina Subregión Arqueológica Diquís, la cual incluye el sureste costarricense; Pacífico Sur y el Caribe Sur, este último incluyendo la Cordillera de Talamanca.

Imagen 4. Regiones Arqueológicas de Costa Rica: A. Subregión Guanacaste de la Región Gran Nicoya. B. Región Central (1. Subregión Central-Pacífica, 2. Subregión Caribe, 3. Subregión Llanuras del Norte). C. Subregión Diquís de la Región Gran Chiriquí. Fuente: Fernández, 2020, p. 2, editada a partir de Corrales, 2001, p. 7.

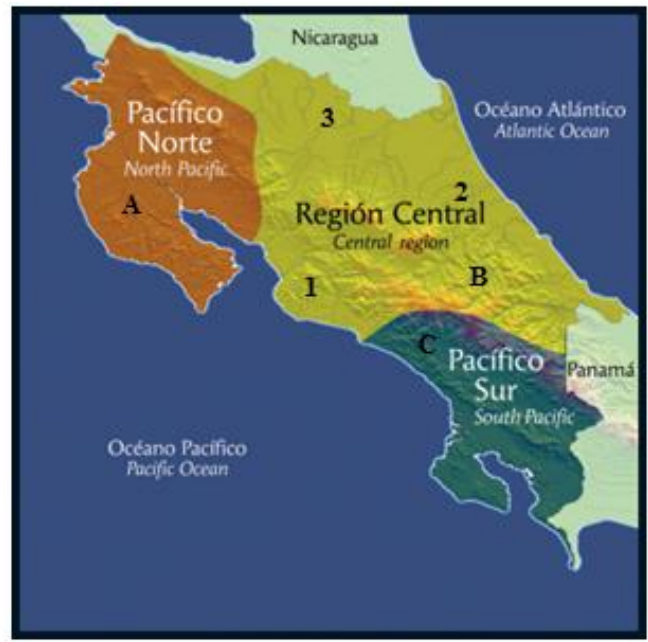

Como tercer concepto se encuentra el de territorio, construyéndose su definición a partir del concepto de territorialidad, definida cómo:

\footnotetext{
${ }^{4}$ Texto original: "of the pottery, settlements and burials"
} 
las acciones desarrolladas por varios agentes sociales en una determinada área geográfica y en un dado momento histórico. Las acciones son producidas por las diferentes relaciones establecidas entre los agentes [...] En esas relaciones están incluidos [...] los elementos culturales, tales como la lingüística, la moral, la ética, la religión, en fin, un conjunto complejo de patrones de comportamiento, dado por las creencias, las instituciones y los valores espirituales y materiales que son transmitidos colectivamente y que caracterizan a una dada sociedad $^{5}$ (MACHADO, 1997, p. 28, traducción propia).

Por lo tanto, según dicha cita textual y Claval (1999), el territorio puede definirse como el resultado de la apropiación o sentido de pertenencia de una sociedad hacia un espacio o espacios delimitados. Dicha apropiación puede surgir a partir del conjunto de características físico-geográficas y culturales que provee el territorio, las cuales dan paso a la instalación de los asentamientos humanos de las diversas poblaciones a lo largo de la historia.

Ejemplo de la aplicación del concepto de territorio vinculado al concepto de territorialidad para el análisis de la evidencia arqueológica, se muestra por medio del ya expuesto sitio Monumento Nacional Guayabo (C-362 MNG) (ver imagen 1 y 2). El mismo, según Aguilar (1972) y (Fonseca, 1992 apud Herrera y Arias, 2016), permite inferir que posiblemente durante un lapso prolongado ha presenciado diversas territorialidades identificadas por medio de la presencia de elementos espaciales como montículos, basamentos, calzadas, estructuras arquitectónicas, entre otros, así como de elementos artefactuales como la cerámica. Los mismos en conjunto permiten acercarnos a proponer que una o varias poblaciones se asentaron en dicho espacio y desarrollaron un vínculo con el mismo por medio de la territorialidad ejercida a partir de diversas prácticas espaciales, mismas que permitieron la consolidación de una sociedad/es organizada/s jerárquicamente.

Como cuarto concepto se encuentra el lugar. Para analizar el espacio, dicho concepto implementa significativamente la variable simbólica, por lo que, según Suess y Ribeiro (2017), el lugar se construye en el espacio a partir de las vivencias de las sociedades, estas desarrolladas según sus intereses y necesidades. De acuerdo a lo anterior, el espacio se "transforma en lugar en la medida en que lo conocemos mejor y le dotamos de valor"6 (FERREIRA, 2000, p. 67, traducción propia), siendo parte del reflejo de la memoria colectiva de la humanidad.

Por lo tanto, el lugar se identifica a partir de la evidencia arqueológica en la medida en que la misma permita reconstruirlo. Lo anterior, según los atributos que presente el espacio, los cuales sean indicadores de

\footnotetext{
5 Texto original: “às ações desenvolvidas por vários agentes sociais em uma determinada área geográfica e em un dado momento histórico. As ações são produzidas pelas diferentes relações establecidas entre os agentes [...]. Nessas relações estão incluídos [...] os elementos culturais tais como lingüística, a moral, a ética, religião, enfim, o conjunto complexo de padrões de comportamento, dado pelas crençias instituições e valores espirituais e materiais que são transmitidos coletivamente e que caracterizam uma dada sociedade".

6 Texto original: "transforma-se em lugar á medida que o conhecemos melhor e o dotamos de valor".
} 
un posible valor simbólico asignado por las poblaciones antiguas. Destacando que, debido a la naturaleza de la evidencia arqueológica, difícilmente será posible saber qué significado o significados tenía un determinado lugar, por lo que solamente es posible inferir con certeza que existieron significados para el mismo, dotándolo así de valor simbólico. Esto se ejemplifica a partir del sitio arqueológico Finca 6 (P-254), localizado en el Delta del Diquís, sector sureste de Costa Rica (ver imagen 5 y 6). Dentro de las estructuras arquitectónicas que presenta, según Corrales (2015), se ha identificado un montículo dentro del cual se ubican contextos funerarios, permitiendo esto inferir que posiblemente fueron lugares debido a que su uso para el enterramiento humano puede indicar un valor simbólico asignado de manera colectiva por las poblaciones antiguas.

Imagen 5. Localización aproximada del sitio Finca 6 (P-254). Fuente: Corrales, 2015, p. 10.

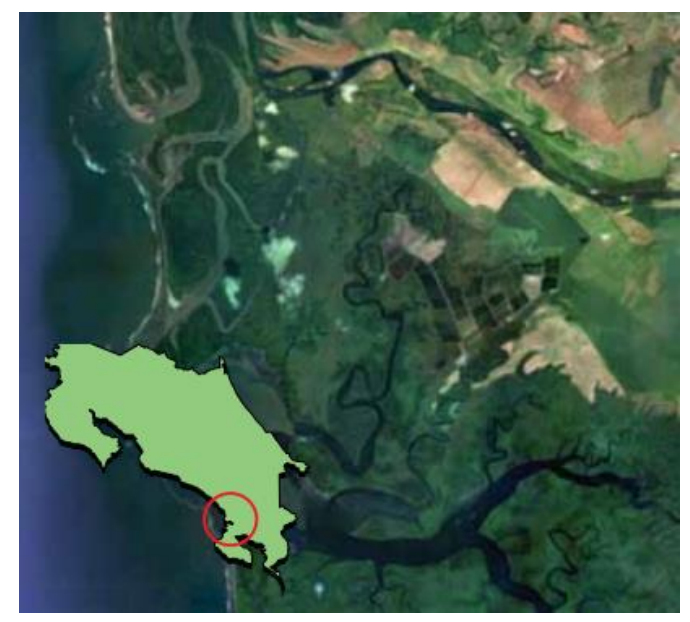

Imagen 6. Montículo funerario en el sitio Finca 6 (P-254). Fuente: Corrales, 2015, p. 32.

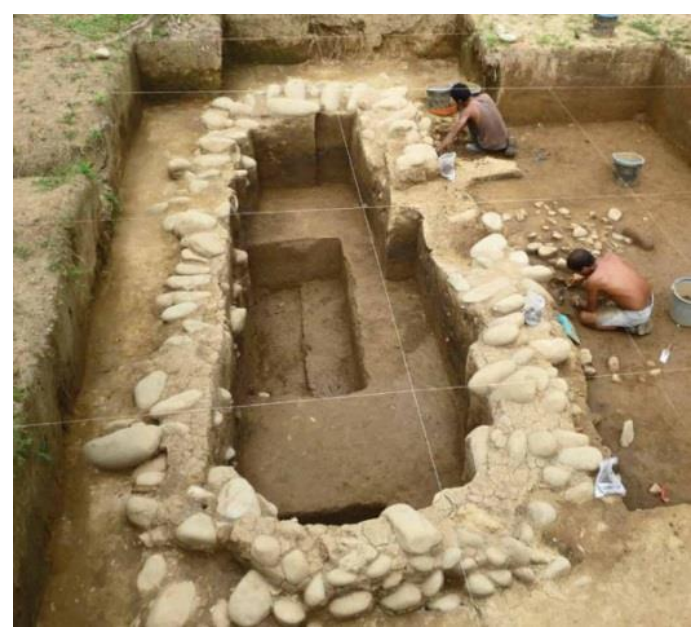


Por último, cabe resaltar el concepto de paisaje, definiéndose como "un espacio o lugar [...] que se contempla y no se está físicamente dentro de él en el momento de la contemplación" (SÁNCHEZ, 2012, p. 12). A partir de esta definición, es posible vincular los conceptos de paisaje y lugar para el análisis de la evidencia arqueológica, debido a que la misma al observarse dentro del paisaje permite reconstruir los posibles lugares que formaron parte de los sitios arqueológicos. Ejemplo de esto lo demuestra el sitio Nuevo Corinto (L-72-NC) (ver imagen 7), localizado en el Caribe costarricense. Esto debido a que, según Gamboa (2016), desde el interior del sitio se visualizan a una distancia aproximada de $26 \mathrm{Km}$ lineales los volcanes Turrialba e Irazú (ver imagen 8). Esto, según la misma autora, permite proponer que posiblemente la localización del sitio fue intencional en relación al paisaje que reflejan ambos volcanes, pudiendo los mismos tener un valor simbólico para las poblaciones antiguas que vivieron en el sitio.

Imagen 7. Localización del sitio Nuevo Corinto (L-72-NC). Fuente: Gamboa, 2016, p. 2.

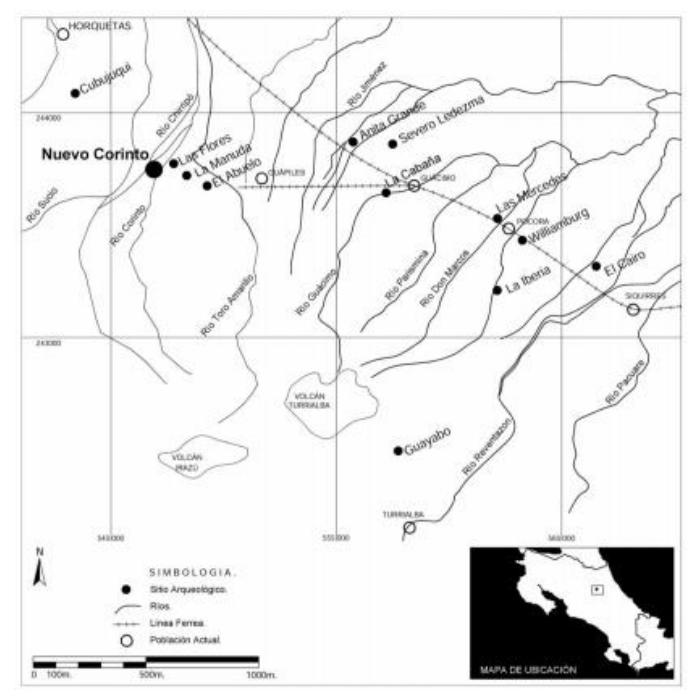

Imagen 8. Paisaje desde el sitio Nuevo Corinto (L-72-NC) hacia el volcán Turrialba e Irazú. Vista desde el montículo 1 en dirección sureste. Fuente: Gamboa, 2016, p. 65.

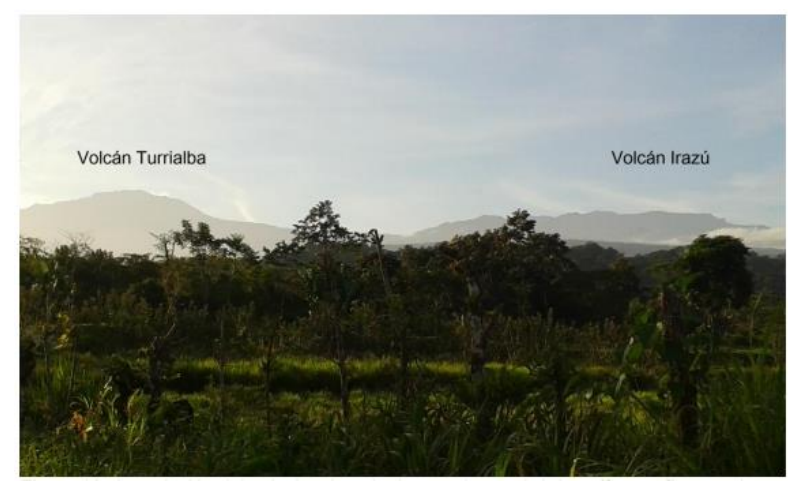


Gestión comunitaria desde la Geografía del Turismo

La Geografía del Turismo, según Morera y Miranda (2016) y Alvarado (2019), se define como una subdisciplina de la Geografía la cual construye su objeto de estudio a partir del análisis territorial del turismo, consecuentemente, su respaldo teórico y metodológico permite gestionar el Patrimonio Cultural -el cual incluye el Patrimonio Arqueológico- en pro de su conservación y preservación a partir de la puesta en valor de los diversos recursos turísticos que presente.

En base a lo anterior, se hace viable considerar las territorialidades identificadas para las poblaciones antiguas como insumo para la gestión comunitaria del Patrimonio Arqueológico, debido a que se pueden concretizar como un recurso turístico de valor cultural significativo, el cual al ser expuesto al público de manera responsable dará paso a su resguardo, funcionando como un medio por el cual se puede inculcar el valor del Patrimonio Cultural. Es decir, dicha puesta en valor es un medio por el cual las personas se pueden aproximar para la comprensión de las poblaciones en su conformación histórica, de entender las diversas manifestaciones que la humanidad ha construido a través del tiempo para sobrevivir y convivir.

Ahora bien, calando en el concepto de gestión, según (Arroyo, 2016 apud Arroyo, 2017), este es un proceso planificado y dirigido para un manejo integral del sistema ambiental, teniendo como objetivo principal alcanzar el desarrollo de relaciones vinculantes y equilibradas con el fin de proteger y trabajar de manera sostenible las distintas variables que construyen dicho sistema. Puntualizando en la gestión comunitaria, Colmenares (2011) la define como aquellos sujetos o actores claves que pertenecen y se sienten parte de la comunidad, los cuales se comprometen para transformar los entornos inmediatos, aprovechando al máximo los recursos que el sistema ambiental les provee con el fin de alcanzar un bien común y mejores condiciones de vida. Siendo el proceso de gestión comunitaria exitoso si los y las participantes "se sienten parte del proceso como producto de sus propias reflexiones, cuestionamientos y miradas de la realidad" (COLMENARES, 2011, p. 32).

En términos arqueológicos, según Colmenares (2011) y Arroyo (2017), el incluir la variable comunidad dentro de la gestión es indispensable debido a que es la propia comunidad, como sujeto activo contribuyente para transformar la realidad, la que debe de trabajar de manera organizada con el fin de obtener beneficios culturales (identidad histórico y socio-cultural), es decir, que la comunidad a partir de su propia labor construya su apropiación de los elementos socio-culturales del Patrimonio Arqueológico. Para este punto, es importante recordar, según Colmenares (2011), que la Arqueología como Ciencia Social tiene el deber de fomentar en las sociedades un pensamiento crítico y consciente ante las realidades que vivimos, promover el desarrollo de ideas creativas, potenciales e innovadoras, las cuales repercutan en la transformación dinámica y positiva para el diario vivir. 


\section{Propuesta metodológica}

A continuación se desarrolla la propuesta metodológica la cual permite la identificación de las territorialidades de las poblaciones antiguas que habitaron en un sitio arqueológico determinado. La misma se segmenta en cuatro apartados, siendo los mismos: la selección del sitio arqueológico, la búsqueda de referencias bibliográficas, el análisis espacial y el análisis crítico. Este último a su vez se divide en cuatro apartados, siendo: la construcción de los indicadores arqueológicos, las propuestas de las categorías analíticas desde la Geografía, la vinculación entre los indicadores arqueológicos y las categorías analíticas geográficas, y el análisis para la identificación de las territorialidades.

\section{A. Selección del sitio arqueológico}

El sitio arqueológico debe contar como mínimo con las siguientes características:

A.1. Debe de existir un interés y compromiso por parte de la comunidad y de sus actores claves, así como de las instituciones que velan, según la legislación, por su protección. Esto podrá dar paso a la identificación de otros actores los cuales estén interesados en contribuir en beneficio de la conservación del Patrimonio Arqueológico. Lo anterior es clave, debido a que de esto depende el éxito de la implementación de un proyecto que vele por el resguardo de dicho patrimonio. Asimismo, se debe de considerar la legislación vigente en relación al Patrimonio Arqueológico y al Turismo, así como de la previa identificación de la condición legal del terreno al cual pertenece el sitio arqueológico, es decir, si el mismo es de propiedad privada, de carácter comunitario o si está una entidad estatal a su cargo.

A.2. Debe de ser accesible en relación a su localización, tanto para las personas de la comunidad como para las posibles visitas de manera externa.

A.3. Para identificar las territorialidades de las poblaciones antiguas es necesario que existan investigaciones científicas específicas para el sitio arqueológico y en otros a su alrededor, esto con el fin de ampliar la capacidad de su análisis territorial en relación a otros espacios. Las mismas deben de tener principalmente un énfasis arqueológico, sin embargo, aquellas de carácter histórico serán de utilidad, destacando que esto no limita la utilización de otras investigaciones las cuales respondan a otras disciplinas científicas, siempre y cuando aporten significativamente a la comprensión de las antiguas poblaciones que habitaron y/o visitaban el sitio. Lo anterior se debe a que del resultado de dichas investigaciones dependerán los datos los cuales permitan identificar las posibles territorialidades de las poblaciones o comunidades antiguas. 
Cabe agregar que para los puntos A.1 y A.2 es imprescindible la realización del trabajo de campo para la obtención de la información. El mismo funciona como un primer acercamiento para la identificación de los actores claves dentro de la comunidad, así como del inicio del trabajo en conjunto con la misma.

Asimismo, para dichos puntos es recomendable la implementación de un Sistema de Información Geográfica (SIG) preferiblemente de software libre, esto debido al acceso, por lo que se puede considerar la implementación de QGIS (se debe contar con los respectivos shapes de la información geográfica base, como curvas de nivel, red hídrica, red vial, etc.). También, la utilización de las plataformas gratuitas de Google Maps ${ }^{8}$ o Google Earth ${ }^{9}$ son de gran utilidad debido a que funcionan como una herramienta la cual permite tener un primer acercamiento con el espacio de estudio como preámbulo a la visita de campo y posibilitando la visualización de rutas de acceso, topografía, entre otros.

\section{B. Búsqueda de referencias bibliográficas}

Habiendo identificado el sitio arqueológico, lo que corresponde realizar es la búsqueda bibliográfica de las producciones científicas e informes arqueológicos que se han desarrollado para el mismo, procurando acercarse al mayor número de ellas, siendo lo idóneo contar con su total, por lo que se amerita su exhaustiva búsqueda en los diversos centros de documentación y bases de datos pertinentes.

Esto es un respaldo teórico-conceptual, el cual permite justificar, según Bunge (1960), lo más fidedignamente los resultados de la identificación de las territorialidades de las poblaciones de la antigüedad, contribuyendo esto a alejar lo más que se pueda un tratamiento subjetivo al momento de analizar la información. En concordancia con lo anterior, para abordar de forma clara y precisa los datos recopilados y aportar a una mejor organización, es recomendable construir una base de datos la cual incluya el nombre del autor(a), el año, el título, la institución que contribuyó a su consolidación, el tipo de producción y la referencia bibliográfica, entre otros. A modo de ejemplo se presenta la Tabla 1:

Tabla 1. Ejemplo de organización de producciones científicas e informes de inspección arqueológica - sitio arqueológico Nuevo Corinto (L-72NC), Limón, Costa Rica. Fuente: Elaboración propia, 2020.

\begin{tabular}{|c|c|c|c|c|c|}
\hline Autora & Año & Título & Institución & Tipo & Referencia bibliográfica \\
\hline $\begin{array}{l}\text { Silvia Sanabria } \\
\text { Hernández }\end{array}$ & 2015 & $\begin{array}{l}\text { La lítica del Sitio Nuevo } \\
\text { Corinto (L-72NC): un } \\
\text { acercamiento a la } \\
\text { dinámica socio- } \\
\text { económica de su }\end{array}$ & $\begin{array}{l}\text { Universidad } \\
\text { de Costa } \\
\text { Rica - } \\
\text { Escuela de }\end{array}$ & $\begin{array}{c}\text { Tesis } \\
\text { Maestría }\end{array}$ & $\begin{array}{l}\text { SANABRIA, Silvia. La lítica del Sitio Nuevo } \\
\text { Corinto (L-72NC): un acercamiento a la } \\
\text { dinámica socio-económica de su producción } \\
\text { en las Fases la Selva-La Cabaña (300 n.e - } \\
1550 \text { n.e). Disertación (Maestría en }\end{array}$ \\
\hline
\end{tabular}

\footnotetext{
${ }^{7}$ https://qgis.org/es/site/

${ }^{8} \mathrm{https} / / / \mathrm{www}$. google.com/maps

${ }^{9}$ https://www.google.com/intl/es-419/earth/
} 
producción en las Fases

la Selva-La Cabaña (300

n.e -1550 n.e)
Antropologí

a

Antropología con énfasis en Arqueología) Facultad de Ciencias Sociales, Universidad de Costa Rica, San José, 2015.

\section{Análisis espacial}

No se puede prescindir del análisis de los elementos físico-geográficos presentes en el sitio arqueológico determinado para identificar y analizar las posibles territorialidades de las poblaciones antiguas que lo habitaron o transitaron. Según lo anterior, se torna necesario recurrir al análisis espacial, el cual se define como "una serie de técnicas matemáticas y estadísticas aplicadas a los datos distribuidos sobre el espacio geográfico" (BUZAI, 2015, p. 57), requiriéndose del uso de un SIG.

En este caso, al contar con un SIG y la respectiva información geográfica base (formatos shapes o fotografías aéreas, etc.), se posibilita el análisis espacial de los cinco elementos propuestos por Buzai (2015): Localización, Distribución Espacial, Asociación Espacial, Interacción Espacial y Evolución Espacial. De esta forma, se hace evidente y viable el análisis de aspectos como la hidrología, la topografía, la geomorfología, los tipos de suelo, el uso del suelo actual, entre otros. Al destacar estos elementos mediante la elaboración de cartografía temática se contribuye significativamente al análisis y a su posterior ilustración.

Sin embargo, cabe plantearse la pregunta; ¿por qué se requiere implementar dicho análisis espacial para la identificación y el análisis de las territorialidades de los pueblos antiguos? Recordando a Bunge (1960), la Ciencia debe de aproximarse a la objetividad en su construcción y por ende en sus resultados, por lo tanto, implementar el análisis espacial basado en los SIG complementa la información obtenida a partir de las investigaciones científicas desarrolladas para el sitio de interés, funcionando como un respaldo teórico y metodológico el cual contribuye a justificar los resultados de las posibles territorialidades antiguas.

A modo de ejemplo, a partir del análisis de los elementos físico-geográficos se obtiene información la cual permite aproximarse a comprender parte de la disponibilidad y el aprovechamiento de los recursos naturales accesibles a dichas comunidades o poblaciones antiguas, pudiéndose esto relacionar con las posibles territorialidades desarrolladas en su momento. Lo anterior se completa con trabajo de campo in situ que permite corroborar la información trabajada.

\section{Análisis crítico}

Según Bunge (1960), la Ciencia debe de ser analítica, por lo tanto, dentro de esta propuesta metodológica el análisis es una herramienta indispensable para aproximarnos a la identificación de las posibles 
territorialidades de las poblaciones antiguas que habitaron el sitio arqueológico de interés, este aplicado tanto por medio del SIG, como a los documentos científicos publicados. Para iniciar el desarrollo de este ejercicio de lectura crítica y analítica de dichos documentos, se requiere de contar con la base de datos previamente establecida, esto con el fin de desarrollar los pasos a continuación presentados.

\section{D.1. Construcción de los indicadores arqueológicos}

Inicialmente, se requiere del análisis crítico para la construcción de los indicadores arqueológicos, destacando que se recomienda considerar como indicador a "una expresión [...] observable y verificable que permite describir características, comportamientos o fenómenos de la realidad social" (DEPARTAMENTO NACIONAL DE PLANEACIÓN-COLOMBIA, 2018, p. 6). Los mismos serán dictados por las investigaciones científicas e informes recopilados para cada sitio arqueológico en particular.

A modo de ejemplo, para el sitio arqueológico Agua Caliente (C-35 AC), localizado en Cartago, Costa Rica (ver imagen 9), mediante la aplicación de este ejercicio se identificaron los siguientes indicadores arqueológicos, destacando que no son todos los posibles, según Gutiérrez (1980) (2004), Vázquez (1989) (1998) (2008), Peytrequín y Aguilar (2007), Vázquez y Villalobos (1999), Valerio, Solís y Solís (1986) y Valerio (1989): basamentos, calzadas, cementerios, empedrados, fogones, montículos, espacios públicos, tumbas, cerámica como escudillas y ollas, huesos como dientes y fragmentos de cráneos, cantos rodados, lajas calcáreas e ígneas, petroglifos, fragmentos líticos de esculturas, pedestales y metates, escultura lítica humana denominada "Mamita", orfebrería como un cascabel de oro, caminos, cuentas de vidrio, posibles espacios habitacionales alrededor del sitio, semillas carbonizadas de maíz y frijol y cerámica procedente de otras regiones arqueológicas. Por lo tanto, al obtener todos los indicadores arqueológicos posibles para el sitio arqueológico de interés, lo que corresponde realizar es su colocación en la matriz más adelante presentada. 
Imagen 9. Localización del sitio Agua Caliente (C-35 AC). Fuente: Peytrequín, 2009, p. 33.

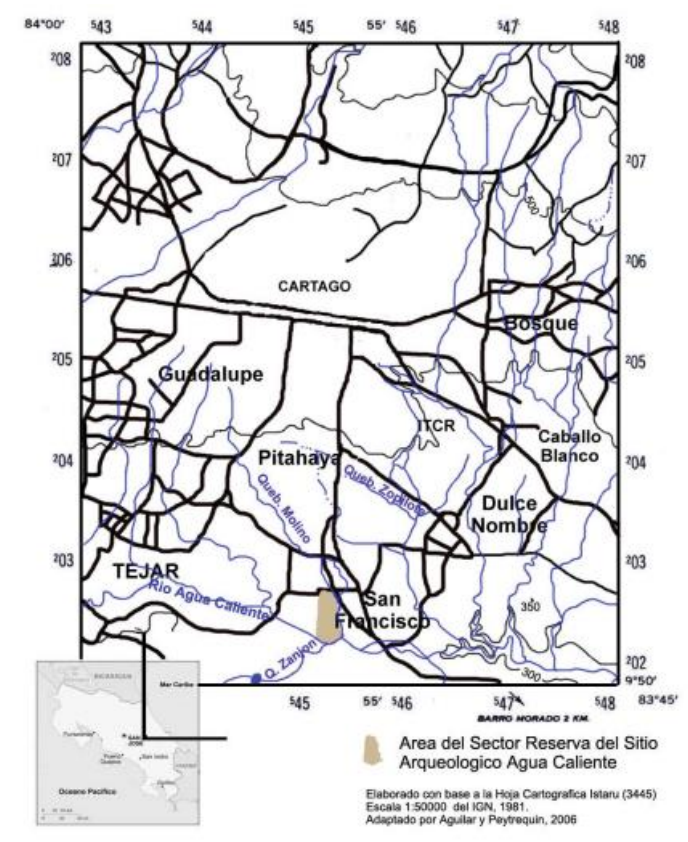

D.2. Propuesta de las categorías analíticas desde la Geografía

Partiendo de la Geografía, las categorías analíticas planteadas para esta propuesta metodológica son el producto de la lectura crítica y analítica de la definición de los conceptos de espacio, región, territorio, paisaje y lugar presentados en el primer apartado. Los mismos, debido a la imposibilidad de abarcar toda la teoría propuesta para su definición, son abordados desde algunos textos provistos por el curso de Geografía e Turismo del Programa de Pós-Graduação em Geografia de la Universidade do Estado do Rio de Janeiro y otros seleccionados por su claridad y precisión al momento de definirlos. En la Tabla 2 se presentan dichos textos:

Tabla 2. Referencias bibliográficas utilizadas para la construcción de las categorías analíticas geográficas. Fuente: Elaboración propia, 2020.

\begin{tabular}{|c|c|c|}
\hline Autor/a & Año & Título \\
\hline Roberto Lobato Côrrea & 2000a & Espaço, um conceito-chave da Geografia \\
\hline Roberto Lobato Côrrea & 2019 & Tempo, espaço e Geografia - um ensaio \\
\hline Roberto Lobato Côrrea & $2000 b$ & Região: um conceito complexo \\
\hline Rógerio Haesbaert & 1999 & Região, diversidade territorial e globalização \\
\hline Mônica Sampaio Machado & 1997 & $\begin{array}{l}\text { Geografia e Epistemologia: Um passeio pelos conceitos de espaço, território e } \\
\text { territorialidade }\end{array}$ \\
\hline Paul Claval & 1999 & Território na transição Pós-Modernidade \\
\hline Luiz Felipe Ferreira & 2000 & $\begin{array}{l}\text { Acepçôes recentes do conceito de lugar e sua importancia para o mundo } \\
\text { contemporáneo }\end{array}$ \\
\hline Ana Fani Alessandri & 2007 & O lugar no/do mundo \\
\hline $\begin{array}{l}\text { Miguel Angelo Ribeiro y Rógerio } \\
\text { Botelho de Mattos }\end{array}$ & s.f. & $\begin{array}{l}\text { Considerações sobre os territórios da prostitução de rua na área central do Rio } \\
\text { de Janeiro }\end{array}$ \\
\hline $\begin{array}{l}\text { Rodrigo Capelle Suess y Antonia da } \\
\text { Silva Samir Ribeiro }\end{array}$ & 2017 & $\begin{array}{l}\text { O lugar na geografia humanista: uma reflexão sobre o seu percurso e questões } \\
\text { contemporâneas - escala, críticas e cientificidade }\end{array}$ \\
\hline Luis Sánchez Ayala & 2012 & El estudio de la geografía \\
\hline Meylin Alvarado Sánchez & 2019 & Un acercamiento a la Geografía del Turismo \\
\hline
\end{tabular}


Por lo tanto, se propone como categorías analíticas provistas desde la Geografía las siguientes: agentes sociales, control socio-espacial, diferencia socio-espacial, ejercicio del poder, fragmentación espacial, sentido de pertenencia, práctica espacial, acción social, manifestación física, apropiación espacial, delimitación espacial, gestión territorial, identidad, memoria colectiva, relaciones ambientales, relaciones sociales, organización territorial, trabajo colectivo, orden simbólico, significado, valor, vivencias, observación, producción y reproducción. Las mismas se debe de colocar en la matriz más adelante presentada.

Resaltando nuevamente que estas son solamente una propuesta y no pretenden ser abordadas de manera fija ni única, todo lo contrario, las mismas están sujetas a modificación y a ser mejoradas por parte de la comunidad científica la cual esté interesada en contribuir dentro de los ejes temáticos a los cuales esta propuesta metodológica pretende aportar. Esto debido a que se debe de recordar que la Ciencia, según Popper (2007), debe de ser progresiva, por lo que requiere de autocorregirse para generar nuevas propuestas de investigación con el fin de contribuir a las necesidades de la sociedad.

\section{D.3. Vinculación entre los indicadores arqueológicos y las categorías analíticas geográficas}

Habiendo recopilado la mayor cantidad posible de datos que permitan proponer los indicadores arqueológicos, el siguiente paso es vincularlos con las categorías analíticas propuestas. Es este sentido, es necesario relacionar los elementos provenientes de ambas disciplinas con el fin de identificar las posibles territorialidades asociadas a las poblaciones antiguas que habitaron o utilizaron el sitio arqueológico en estudio. Es por esto que se propone la construcción de una matriz la cual permita relacionarlos. A continuación se presenta el resultado de una matriz tomando como ejemplo algunos indicadores arqueológicos del sitio Agua Caliente (C-35 AC). Destacando que la misma se debe de completar marcando con una X (o cualquier otro símbolo) si el campo correspondiente al indicador arqueológico se vincula con la categoría analítica geográfica. Esto como base para el siguiente paso. 
Tabla 3. Ejemplo de matriz para la vinculación de los indicadores arqueológicos del sitio Agua Caliente (C-35 AC) y las categorías analíticas geográficas mencionadas. Fuente: Elaboración propia, 2020.

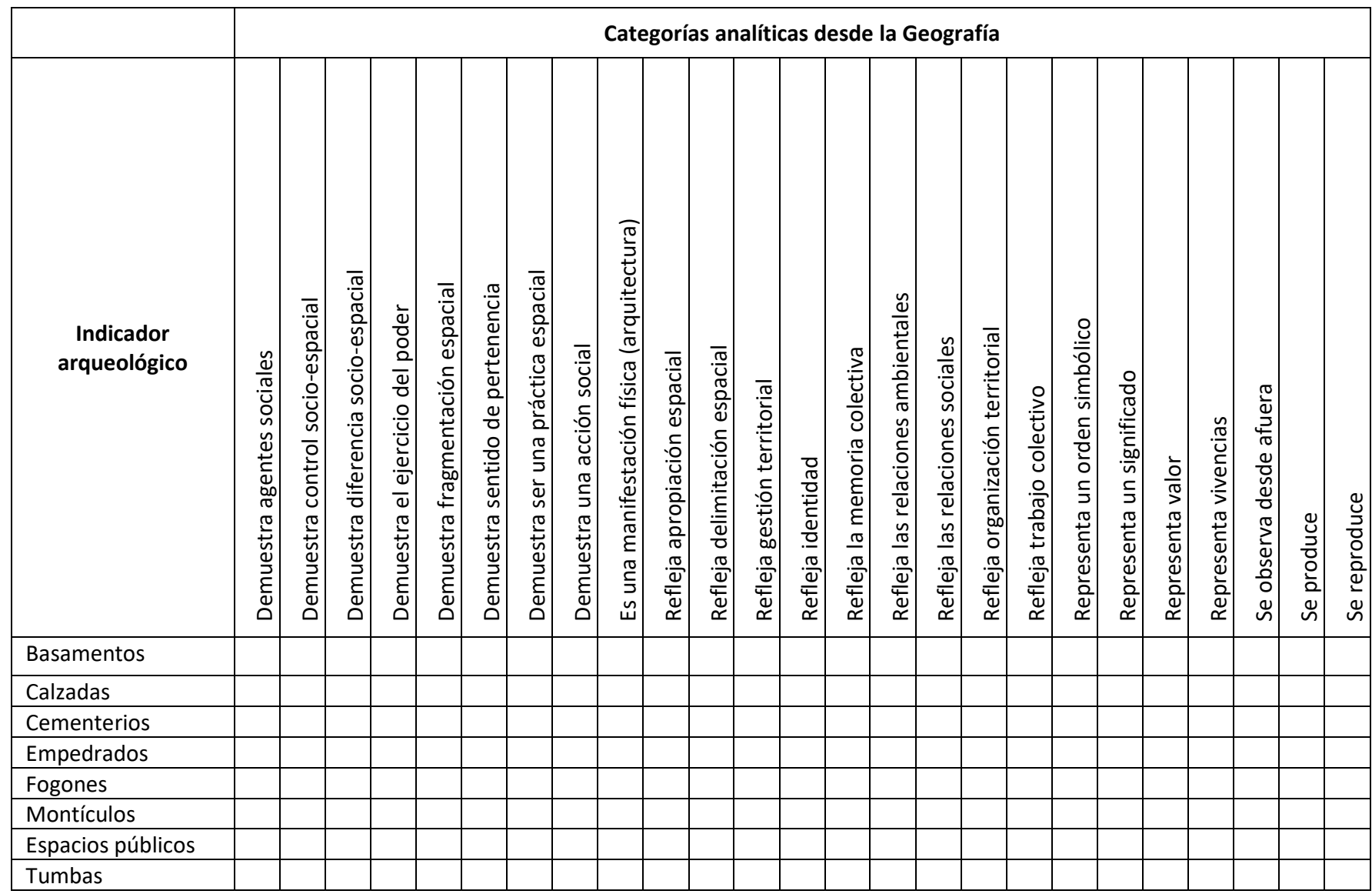

\section{D.4. Análisis para la identificación de las territorialidades}

¿Cómo se pueden identificar las posibles territorialidades de las poblaciones antiguas a partir de la evidencia arqueológica? Antes de responder a esta interrogante, es preferible puntualizar nuevamente el concepto de territorialidad. La misma, basándose en lo propuesto por Machado (1997) y Côrrea (2000a), significa la apropiación por parte de un grupo social a un espacio, transformado en territorio a partir de la creación y ejecución de diversas prácticas espaciales producidas por las relaciones sociales, producto de la necesidad de sobrevivencia y de la convivencia humana. Por lo tanto, las posibles territorialidades se identifican a partir del análisis crítico y justificado de la relación de los indicadores arqueológicos con las categorías analíticas geográficas, es decir, es necesario preguntarnos el ¿por qué se relacionan?, recordando que para ello es fundamental contar con el sustento teórico-conceptual del cual se respaldan. De acuerdo a lo anterior, los resultados obtenidos en la elaboración de la matriz permiten concretar un producto el cual responda a lo que significa lo que desde la geografía se está concibiendo como la territorialidad para las y los antiguos pobladores del asentamiento analizado. 
Asimismo, se debe tener en cuenta la vinculación de los resultados basados en las publicaciones científicas con los obtenidos por medio del desarrollo del análisis espacial de las características físicogeográficas identificadas en el área de estudio, siendo la misma el sitio arqueológico de interés.

\section{REFLEXIONES FINALES}

La síntesis conceptual presentada inicialmente, vinculada a la propuesta metodológica expuesta, permite identificar que el abordaje de la evidencia arqueológica desde la Geografía es viable para acercarnos a la comprensión de las posibles territorialidades de las poblaciones antiguas, consolidándose esto como un insumo teórico-conceptual y práctico para la gestión y resguardo del Patrimonio Arqueológico desde la Geografía del Turismo.

Asimismo, considerando el carácter útil de la Ciencia mencionado por Bunge (1960), la propuesta presentada al considera a ambas disciplinas como parte de las Ciencias Sociales, permite establecer su utilidad en cuanto a que posibilita acercarse a la comprensión de la realidad social en distintos espacios y tiempos, y al ponerlas en práctica permiten fomentar relaciones humanas de convivencia, vinculadas a la estructura social, económica, política y ambiental, aportando así de manera significativa en el desarrollo de relaciones sociales equitativas.

Como recomendación, para desarrollar una propuesta de gestión comunitaria es posible apoyarse en teoría y metodología proveniente de la ciencia Pedagógica, específicamente en la subdisciplina Didáctica. Resaltando que la primera, según Francis (2012), se vincula directamente con la formación intencional en el ámbito educativo, con el fin de transformar el conocimiento de las personas para dar paso a la liberación y desarrollo de análisis crítico ante el entorno que les rodea. Por otra parte, según la misma autora, construye el hecho educativo y lo caracteriza a partir de las necesidades que proporcione su contexto y justifica a la educación como un espacio necesario de intervención social. Por otra parte, según (Flores, 1994 apud Flores et al., 2017), la Didáctica responde a las necesidades educativas de la sociedad, parte de sus funciones es estudiar e intervenir para organizar y favorecer el proceso de enseñanza-aprendizaje.

Con lo anterior, se torna complejo desligar los procesos de enseñanza-aprendizaje con el pretender abordar un sitio arqueológico con una comunidad, por lo que como aportaciones de ambas ciencias se pueden implementar las Estrategias Didácticas para el resguardo del Patrimonio Arqueológico. Las mismas se definen, según Flores et al. (2017), como aquellos procesos o recursos facilitadores que implementa un/una docente o un/una moderador/a, con el fin de alcanzar con éxito los procesos de enseñanza-aprendizaje de manera significativa en los y las estudiantes o comunidad. Esto nos permite reflexionar que la labor pedagógica no 
solamente debe de relacionarse con los contextos de "aula" o instituciones educativas, sino que dicho bagaje científico se debe de aplicar en los diversos contextos en donde las sociedades humanas se desenvuelven.

Por último, al reflexionar en torno a lo anterior, cabe concluir que la Arqueología no se desvincula de la Geografía para abordar la realidad social, debido a que la evidencia con la cual trabaja se encuentra sumergida en el espacio geográfico -físico y social-, por lo que su análisis requiere parte del aporte conceptual y práctico que provee la Geografía para la obtención de resultados con mayor capacidad de análisis.

\section{AGRADECIMIENTOS}

Agradezco profundamente a Meylin Alvarado Sánchez, Olger Vega Espinoza, Gabriela Arroyo Wong, Mónica Aguilar Bonilla y Daniel Avendaño Leadem, por sus observaciones y sugerencias para este trabajo.

\section{REFERENCIAS}

AGUILAR, Carlos. Guayabo de Turrialba: Arqueología de un sitio indígena prehispánico. San José, Costa Rica: Editorial Costa Rica, 1972. 192p.

ALESSANDRI, Ana Fani. O lugar no/do mundo. São Paulo: FFLCH, 2007. 85p.

ALVARADO, Meylin. Un acercamiento a la Geografía del Turismo. In: Geografía e Turismo. Reflexôes Interdisciplinarias. Curitiba: Appris, 2019. p. 73-84.

ARIAS, Ana Cecilia; CHÁVEZ, Sergio. La arqueología ¿Qué es y qué significa? Cultura y poder: Cuadernos de Antropología, n. 10, p. 57- 63, 1999.

ARIAS, Felipe. Sitios musealizados y museos de sitio: notas sobre dos modos de utilización del Patrimonio arqueológico. Museo: Revista de la Asociación Profesional de Museólogos de España, n. 4, p. 39-57, 1999.

ARROYO, Gabriela. La gestión comunitaria como herramienta esencial en la protección y conservación del patrimonio arqueológico. Revista Herencia., v. 30, n. 2, p. 109-120, 2017.

BALDEÓN, Amelia. El Patrimonio Arqueológico. Memoria para el futuro. In: XV CONGRESO DE ESTUDIOS VASCOS. Dinostia: Eusko Ikaskuntza, 2002. p. 21-27.

BEOVIDE, Laura. Concheros en la costa uruguaya del río De La Plata: una aproximación a la explotación y uso de moluscos por las sociedades de fines del holoceno medio. Cuadernos del Instituto Nacional de Antropología y Pensamiento Latinoamericano Series Especiales, v. 1, n. 1, p. 136-148, 2013.

BUNGE, Mario. La ciencia. Su método y su filosofía. Buenos Aires: Siglo XX, 1960. 74p.

BUZAI, Gustavo. Conceptos fundamentales del análisis espacial que sustentan la investigación científica basada en Geotecnologías. In: Geografía, geotecnología y análisis espacial: tendencias, métodos y aplicación. Santiago de Chile: Editorial Triángulo, 2015. $208 p$.

CLAVAL, Paul. Território na transição Pós-Modernidade. GEOgrafhia, v. 1, n. 2, p. 7-26, 1999.

COLMENARES, Ana Mercedes. Investigación-acción participativa: una metodología integradora del conocimiento y la acción. Voces y Silencios: Revista Latinoamericana de Educación, v. 3, n. 1, p. 102-115, 2011.

CONFERENCIA GENERAL DE LA ORGANIZACIÓN DE LAS NACIONES UNIDAS PARA LA EDUCACIÓN, LA CIENCIA Y LA CULTURA, 17a, 1972, París. Textos básicos de la Convención del Patrimonio Mundial de 1972 (Convención sobre la protección del Patrimonio Mundial Cultural y Natural). España: UNESCO, 2006. 241p. 
CORRALES, Francisco. An evaluation of long term cultural change in southrn central america: the ceramic record of the Diquís Archaeological Subregion, Southern Costa Rica. Disertación (Maestría en Arqueología) 2000. Tesis (Ph. D. en Antropología) University of Kansas, Estados Unidos, 2000.

CORRALES, Francisco. Asentamientos Cacicales con Esferas de Piedra del Diquís: Sitio de Patrimonio Mundial. San José, Costa Rica: Museo Nacional de Costa Rica, 2015. 51p.

CORRALES, Francisco. Los primeros costarricenses. San José, Costa Rica: Museo Nacional de Costa Rica, 2001. 85p.

CÔRREA, Roberto Lobato. Espaço, um conceito-chave da Geografia. In: Geografia: conceitos e temas. Rio de Janeiro: Bertrand Brasil, 2000a, pp. 15-47. 352p.

CÔRREA, Roberto Lobato. Região: um conceito complexo. In: Região e organização Espacial. São Paulo: Editora Ática, 2000b.

CÔRREA, Roberto Lobato. Tempo, espaço e Geografia - um ensaio. Revista Brasileira de Geografia, v. 64, n. 1, p. 285-294, 2019.

DEPARTAMENTO NACIONAL DE PLANEACIÓN-COLOMBIA. Guía para la construcción y análisis de indicadores. Bogotá:

Departamento Nacional de Planeación, 2018. 33p.

FERNÁNDEZ, Patricia. Sellos precolombinos: imágenes estampadas de Costa Rica. San José, Costa Rica: Fundación Museos Banco Central de Costa Rica, 2020. 28p.

FERREIRA, Luiz Felipe. Acepçôes recentes do conceito de lugar e sua importancia para o mundo contemporáneo. Revista Território, n. 9, p. 65-83, 2000.

FLORES, Jael; ÁVILA, Jorge; ROJAS, Constanza; SÁENZ, Fernando; ACOSTA Robinson y DÍAZ; Claudio. Estrategias didácticas para el aprendizaje significativo en contextos universitarios. Universidad de Concepción. Concepción, Chile: Unidad de Investigación y Desarrollo Docente, Dirección de Docencia, Universidad de Concepción, 2017. 150p.

FRANCIS, Susan. El conocimiento pedagógico del contenido como modelo de mediación docente. San José, Costa Rica: Coordinación Educativa y Cultural (CECC/SICA), 2012. 120p.

GAMBOA SEGURA, Kendra. Evidencia del cambio social en la arquitectura del sitio Nuevo Corinto L-72-NC, Guápiles, Limón. 2016. Disertación. (Maestría en Antropología con énfasis en Arqueología) - Facultad de Ciencias Sociales, Universidad de Costa Rica, San José, 2016.

GOOGLE EARTH. Sitio web: https://www.google.com/intl/es-419/earth/

GOOGLE MAPS. Sitio web: https://www.google.com/maps

GUTIÉRREZ, Maritza. Agua Caliente, sector Los Pinares. Informe de inspección por denuncia. San José: Departamento de Antropología e Historia, Museo Nacional de Costa Rica, 2004.

GUTIÉRREZ, Maritza. Informe de un cementerio Precolombino en Agua Caliente de Cartago. San José: Departamento de Antropología e Historia, Museo Nacional de Costa Rica, 1980.

GUTIÉRREZ, Maritza. La ictofauna del sitio arqueológico Nacascolo, bahía Culebra, Guanacaste. Vínculos, v. 22, p. 157-187, 1997.

HAESBAERT, Rogério. Região, diversidade territorial e globalização. GEOgrafhia, v. 1, n. 1, p. 15-39, 1999.

HERRERA, Grece; ARIAS, Ana Cecilia. Los petrograbados de Guayabo de Turrialba, Costa Rica: Un acercamiento a su significado. Revista Herencia, v. 29, n. 2, p. 175-204, 2016.

MACHADO, Mônica Sampaio. Geografia e Epistemologia: Um passeio pelos conceitos de espaço, território e territorialidade. GEOUERJ, n. 1, p. 23-30, 1997.

MORERA, Carlos y MIRANDA, Pablo. De la Geografía del Turismo al Análisis Territorial del Turismo: el rastro en Costa Rica. Revista Geográfica de América Central, n. 54, p. 15-43, 2015.

PEYTREQUÍN GÓMEZ, Jeffrey.; AGUILAR BONILLA, Mónica. Agua Caliente (C-35AC): Arquitectura, procesos de trabajo e indicadores arqueológicos de un modo de vida cacical en una aldea nucleada en el Intermontano Central, Costa Rica. 2007. Disertación (Licenciatura en Antropología con énfasis en Arqueología) - Facultad de Ciencias Sociales, Universidad de Costa Rica, San José, 2007.

PEYTREQUín, Jeffrey. Agua Caliente, espacialidad y arquitectura en una comunidad nucleada antigua de Costa Rica. Cuadernos de Antropología, n. 19, p. 31-55, 2009.

POPPER, Karl. Conocimiento objetivo. Un enfoque evolucionista. Madrid: Tecnos, 2007. 235p. 
QGIS. Sitio web: https://qgis.org/es/site/

RIBEIRO, Miguel Ângelo; DE MATTOS, Rogério Botelho. Considerações sobre os territórios da prostitução de rua na área central do Rio de Janeiro. Observatorio Geográfico de América Latina, p. 1-10, s.f.

SÁNCHEZ, Luis. El estudio de la geografía. In: Geografía humana. Conceptos básicos y aplicaciones. Bogotá: Ediciones Uniandes, 2012. 210p.

\section{SANTOS, Milton. Por una nueva geografía. Madrid: Espasa-Calpe, 1990. 257p.}

SUESS, Rodrigo Capelle; RIBEIRO, Antonia da Silva Samir. O lugar na geografia humanista: uma reflexão sobre o seu percurso e questões contemporâneas - escala, críticas e cientificidade. Revista Ecuador, v. 6, n. 2, p. 1-22, 2017.

VALERIO, Wilson. Patrones de asentamiento en Agua Caliente de Cartago. Vínculos, v. 15, n. 1-2, p. 25-44, 1989.

VALERIO, Wilson; SOLÍS, Olman; SOLÍS, Felipe. Agua Caliente de Cartago: del rescate súbito a la investigación a largo plazo. Vínculos, v. 12 , n. 1-2, p. 39-50, 1986.

VÁZQUEZ, Ricardo. Desvío y entubado de las aguas de la quebrada El Molino, sector Playskool del sitio Agua Caliente, Cartago. Informe de inspección arqueológica. San José: Departamento de Antropología e Historia, Museo Nacional de Costa Rica, 1998.

VÁZQUEZ, Ricardo. Hallazgo de una escultura tipo "mamita” en el campo recreativo de la urbanización Cocorí: sitio Agua Caliente (C-35 AC). Informe de inspección. San José: Departamento de Antropología e Historia, Museo Nacional de Costa Rica, 2008.

VÁZQUEZ, Ricardo. Representaciones demográficas y estructuras de la organización social en las prácticas funerarias del sitio Agua Caliente, Cartago. Vínculos, v. 15, n. 1-2, p. 1-23, 1989.

VÁZQUEZ, Ricardo; VILLALOBOS, Gabriela. Importancia y proyecciones de la reserva arqueológica del sitio Agua Caliente, Cartago (Banda oriental de la urbanización Cocorí). San José: Departamento de Antropología e Historia, Museo Nacional de Costa Rica, 1999. 